\title{
The Potent Bone-resorbing Mediator of Actinobacillus actinomycetemcomitans Is Homologous to the Molecular Chaperone GroEL
}

\author{
Alun C. Kirby, ${ }^{\star}$ Sajeda Meghji, ${ }^{\ddagger}$ Sean P. Nair, ${ }^{\star \ddagger}$ Peter White, ${ }^{\star \ddagger}$ Krisanavane Reddi, ${ }^{\ddagger}$ Tatsuji Nishihara, ${ }^{5}$ \\ Keisuke Nakashima," Anthony C. Willis, ' Robert Sim,' Michael Wilson, * and Brian Henderson* \\ *Department of Microbiology and ${ }^{\ddagger}$ Maxillofacial Surgery Research Unit, Eastman Dental Institute for Oral Health Care Sciences, \\ University of London, London, WC1X 8LD, United Kingdom; ${ }^{8}$ Department of Oral Science, National Institute of Health, Tokyo, Japan; \\ "Department of Periodontology, Tokyo Medical and Dental University, Tokyo, Japan; and 'MRC Immunochemistry Unit, Department of \\ Biochemistry, University of Oxford, Oxford, United Kingdom
}

\begin{abstract}
Actinobacillus actinomycetemcomitans is a Gram-negative bacterium implicated in the pathology of localized juvenile periodontitis, a condition involving rapid destruction of alveolar bone. We have established that gentle extraction of this bacterium in saline releases a proteinaceous fraction (which we have termed surface-associated material [SAM]) which has potent osteolytic activity in the murine calvarial bone resorption assay. Fractionation of the SAM has now revealed that activity is associated with a $62-\mathrm{kD}$ protein. This bone-resorbing activity can be blocked by a monoclonal antibody (raised to the whole bacterium) that is claimed to recognize a protein homologous to the Escherichia coli molecular chaperone GroEL. Purification of this bone-resorbing protein to homogeneity has been achieved by a combination of anion exchange, gel filtration, and ATP-affinity chromatography and the $\mathbf{N H}_{\mathbf{2}}$-terminal sequence shows $>95 \%$ homology to $E$. coli GroEL. This GroEL homologue is found in the SAM of A. actinomycetemcomitans but is not found in the osteolytically active SAM from other Gramnegative or Gram-positive bacteria. The GroEL protein from $E$. coli, but not from Mycobacterium tuberculosis and Mycobacterium leprae, also showed activity in the bone resorption assay. We believe this to be the first observation that a molecular chaperone has the capacity to stimulate the breakdown of connective tissue. (J. Clin. Invest. 1995. 96:1185-1194.) Key words: periodontal disease - chaperonin 60 • heat shock proteins • bone resorption

\section{Introduction}

The chronic inflammatory periodontal diseases (CIPDs) ${ }^{1}$ are the most prevalent of the persistent inflammatory diseases of

Address correspondence to Professor Brian Henderson, Maxillofacial Surgery Research Unit, Eastman Dental Institute for Oral Health Care Sciences and University College Hospital London, University of London, 256 Gray's Inn Road, London WC1X 8LD, United Kingdom. Phone and FAX: 0171-915-1190.

Received for publication 15 November 1994 and accepted in revised form 25 May 1995.

1. Abbreviations used in this paper: CIPD, chronic inflammatory periodontal disease; LJP, localized juvenile periodontitis; SAM, surfaceassociated material.

J. Clin. Invest.

(C) The American Society for Clinical Investigation, Inc. 0021-9738/95/09/1185/10 \$2.00

Volume 96, September 1995, 1185-1194 humans. They are characterized by inflammation of the gingivae and the resorption of the alveolar bone supporting the teeth, a process which can lead to tooth loss. Evidence strongly suggests that the presence of certain Gram-negative oral bacteria in periodontal pockets (which are formed between the gum and the tooth in this disease) is a major factor in the development of tissue pathology, and this relationship has been best documented with the bacterium Actinobacillus actinomycetemcomitans and its role in localized juvenile periodontitis (LJP) $(1,2)$. However, the precise mechanisms responsible for the resorption of alveolar bone in this disease remain to be established. Bacteria may invade the periodontal tissues (3), but the accepted paradigm is that the resorption mechanism involves factors released by the bacteria, which either directly stimulate bone breakdown or generate the synthesis and/or release of osteolytic mediators within host tissues $(3,4)$. These mediators may include prostanoids and/or certain proinflammatory cytokines such as IL-1 and TNF $\alpha$, all of which have potent osteolytic activity.

Using a simple saline extraction procedure we have isolated a fraction from the surface (termed surface-associated material [SAM]) of a number of bacteria implicated in the pathology of the CIPDs. This fraction contains the capsule and other components loosely associated with the outer membrane of the bacteria, and electron microscopic examination of extracted organisms demonstrates the removal of extracellular material but fails to show evidence of cell lysis $(5,6)$. In the case of A. actinomycetemcomitans this highly soluble fraction consists largely of protein $(>60 \%)$ and an extremely small amount of LPS (6). There is now evidence that the SAM is shed by oral bacteria in situ, being found on the tooth roots of extracted teeth from patients with CIPD (7). The SAM from A. actinomycetemcomitans, has a number of biological activities including inhibition of cellular proliferation (8) and of collagen synthesis (9). However, its most potent action is the stimulation of murine calvarial bone breakdown, with activity being seen at concentrations as low as $1 \mathrm{ng} / \mathrm{ml}(5,6)$. In this assay the SAM is $10-100$ times more potent than the LPS prepared from the same organism (5). Thus, the active moiety in this fraction could be a major factor in the pathogenesis of bone resorption in LJP. A number of bacterial components including LPS, teichoic acids, muramyl dipeptide, and certain protein fractions have been shown to be capable of stimulating bone resorption (10-13). However, with the possible exception of a toxin from the bacterium Pasteurella multocida (14), the SAM from A. actinomycetemcomitans is the most potent bacterial osteolytic agent reported. As described in this paper we have established that the active moiety is a 62 $\mathrm{kD}$ protein and thus its molar potency is in the picomolar range, similar to that reported for potent osteolytic cytokines such as IL-1 or TNF. 
We have now isolated this osteolytic protein by use of anion exchange, gel filtration, and affinity chromatography and have determined its $\mathrm{NH}_{2}$-terminal sequence. Care has been taken to exclude the possibility that LPS is contributing to the osteolytic activity of this protein. Isolation was aided by the use of monoclonal antibodies which neutralize the bone-resorbing activity of the SAM. These studies have established that the osteolytically active protein of $A$. actinomycetemcomitans is a member of the GroEL family of molecular chaperones.

\section{Methods}

Growth of bacteria. A. actinomycetemcomitans NCTC 9710 was grown on brain-heart infusion agar (Oxoid, Basingstoke, United Kingdom) at $37^{\circ} \mathrm{C}$ in a $\mathrm{CO}_{2}$-enriched atmosphere for $3 \mathrm{~d}$. Eikenella corrodens NCTC 10596 and Porphyromonas gingivalis W50 were grown under anaerobic conditions at $37^{\circ} \mathrm{C}$ in a medium consisting of brain-heart infusion agar supplemented with 0.375 grams/liter cysteine- $\mathrm{HCl}$ (BDH), 0.25 grams/ liter haemin (BDH), and 0.05 grams/liter menadione (BDH Poole, Dorset, United Kingdom). Escherichia coli $Y 1090$ was grown on Luria Bertani agar, consisting of 10 grams/liter Bacto-Tryptone (Difco Laboratories Inc., Detroit, MI), 5 grams/liter bacto-yeast extract (Difco), and 5 grams/liter $\mathrm{NaCl}$ (Sigma Immunochemicals, St. Louis, MO), for $24 \mathrm{~h}$ under aerobic conditions at $37^{\circ} \mathrm{C}$. Staphylococcus aureus (Oxford strain NCTC 6571) was grown under aerobic conditions on brain-heart infusion agar at $37^{\circ} \mathrm{C}$ for $48 \mathrm{~h}$.

Extraction of SAM. All cells were harvested in sterile saline, centrifuged, washed briefly in saline, and lyophilized. SAM was removed from the various bacteria by gentle saline extraction as described (5). Briefly, lyophilized bacteria were resuspended in sterile saline and stirred gently at $4^{\circ} \mathrm{C}$ for $1 \mathrm{~h}$. The bacteria were removed by centrifugation and the soluble SAM was dialyzed extensively against distilled water and lyophilized. The protein content of the SAM was determined by the method of Lowry et al. (15), the carbohydrate content by the method of Dubois et al. (16), and the nucleic acid content by absorption at $260 / 280 \mathrm{~nm}$. The LPS content was measured by use of a commercial chromogenic Limulus amebocyte lysate assay (Pyrogent, Byk-Mallinckrodt, London, United Kingdom) according to the manufacturer's instructions. In all studies the activity of the SAM was related to dry weight.

Monoclonal antibodies. Hybridomas were raised in Balb/c mice immunized with A. actinomycetemcomitans ATCC (\#43718) whole cells (17). Briefly, spleen cells from intracutaneously immunized and intraperitoneally boosted mice were fused with SP2/0-Ag-14 myeloma cells. Hybridomas were screened by ELISA for immunoglobulin secretion and antibody producers were cloned by limiting dilution. Three of these, P1, P2, and P3, secreted antibodies which have been shown to be specific for $81-, 62-$, and $62-\mathrm{kD}$ proteins, respectively, and this was confirmed by Western blotting against SAM from A. actinomycetemcomitans NCTC 9710 . Hybridoma cells secreting mAbs P1, P2, and P3 were grown in Iscoves modified Dulbecco's medium (Gibco Laboratories, Grand Island, NY) containing 10\% Seraclone FCS (Sera-Lab Ltd., Crawley Down, United Kingdom) at $37^{\circ} \mathrm{C}$ in an atmosphere of $5 \% \mathrm{CO}_{2} /$ air. When cell death began to occur, the cells were removed by centrifugation and the supernatant was collected. This was filtersterilized and stored at $-20^{\circ} \mathrm{C}$ until needed. Monoclonal antibody A5 was raised against the SAM extracted from A. actinomycetemcomitans NCTC 9710 . Balb/c mice were immunized subcutaneously and boosted intravenously. Hybridoma cells were produced as described above and screened by ELISA against SAM. mAb A5 has been shown to be specific for a $66-\mathrm{kD}$ protein component of the SAM by Western blot. Hybridoma cells secreting A5 were cultured in RPMI 1640 medium (Gibco Laboratories) containing $10 \%$ FCS (Sera-Lab). Tissue culture supernatant was collected as described above. All mAbs were of the IgG1 subclass.

All four mAbs were purified from their respective tissue culture supernatants using a protein A column (Sepharose CL 4B-linked pro- tein A; Bio Rad Laboratories, Hercules, CA) (18) and dialyzed extensively against PBS.

Murine calvarial bone resorption assay. Bone resorption was quantified by measuring calcium release from MF1 strain mouse calvaria in vitro (19). In some experiments the LPS-unresponsive strain $\mathrm{C} 3 \mathrm{H} / \mathrm{HeJ}$ was used. Calvaria were removed from 5-d-old mice and halved, with each half cultured separately on stainless steel grids. Calvaria were cultured in 30-mm dishes with $1.5 \mathrm{ml}$ of BGJ medium (Flow Laboratories Inc., McLean, VA) containing 5\% heat-inactivated rabbit serum (Gibco Laboratories) and $5 \mathrm{mg} / 100 \mathrm{ml}$ ascorbic acid (Sigma Immunochemicals). The calvaria were treated in groups of five. After $24 \mathrm{~h}$, the media were replaced with media containing the test substances. PGE $_{2}$ at $1 \mu \mathrm{M}$ was used as a positive control in all assays (except those in which the $\mathrm{C} 3 \mathrm{H} / \mathrm{HeJ}$ strain mice were used, when IL-1 $\alpha$ was used) to show that the bone was responsive to osteolytic mediators. Calvaria were cultured for a further $48 \mathrm{~h}$ before calcium release was measured by automated colorimetric analysis (20). The significance of the results was calculated using Student's $t$-test. Batches of SAM were assayed for bone resorbing activity over the concentration range $10 \mathrm{ng} / \mathrm{ml}$ to 10 $\mu \mathrm{g} / \mathrm{ml}$.

To assess the capacity of mAbs to inhibit the bone resorbing activity of the SAM, individual mAbs were added to calvarial cultures stimulated to resorb by the presence of $1 \mu \mathrm{g} / \mathrm{ml} \mathrm{SAM}$ or $1 \mu \mathrm{g} / \mathrm{ml} \mathrm{LPS} \mathrm{from} A$. actinomycetemcomitans. Each antibody was used at various dilutions to determine its inhibitory dose response. Nonspecific mouse immunoglobulin G, containing all four IgG subclasses (Sigma Immunochemicals), was used as a control in all the antibody studies. To determine if the mAbs were able to deplete the SAM of its osteolytic activity, solutions of the SAM were incubated with an excess $(1: 10 \mathrm{wt} / \mathrm{wt})$ of antibody P3 or with nonspecific mouse IgG (Sigma Immunochemicals), or with mAb A5 overnight at $4^{\circ} \mathrm{C}$ with constant mixing. Antibody, along with any bound antigen, was then removed by the addition of $S$. aureus (Cowan strain) heat-killed/formalin-fixed whole cells (Sigma Immunochemicals) for $1 \mathrm{~h}$ at room temperature, again with constant mixing, followed by centrifugation and filter sterilization. The depleted fraction was added to the calvarial assay at 1 or $10 \mu \mathrm{g} / \mathrm{ml}$ and activity was compared with that of untreated SAM. SAM was also directly incubated with $S$. aureus at room temperature for $1 \mathrm{~h}$ to control for any possible nonspecific binding events between bacteria and SAM, this treated fraction being tested in the bone resorption assay.

To determine if LPS was contributing to the osteolytic activity of the SAM, the crude and purified GroEL preparations or LPS from A. actinomycetemcomitans were exposed to heat $\left(100^{\circ} \mathrm{C}\right.$ for $\left.30 \mathrm{~min}\right)$ or trypsin ( $0.25 \%$ trypsin; Sigma Immunochemicals) for $1 \mathrm{~h}$ followed by excess (threefold molar excess) of soya-bean trypsin inhibitor (Sigma Immunochemicals ). Control cultures exposed to the trypsin/trypsin inhibitor complex showed no increase in bone resorption, and the formed complex when added to bone cultures stimulated with $\mathrm{PGE}_{2}$ did not inhibit osteolysis. Polymyxin B $(20 \mu \mathrm{g} / \mathrm{ml})$ was also added to bone cultures stimulated with either SAM or LPS from A. actinomycetemcomitans to determine if it could inhibit bone resorption.

SDS-PAGE. The components of the SAM were analyzed by SDSPAGE using $12 \%$ gels according to the method of Laemmli (21). Samples were diluted 1:1 with sample buffer and boiled for 5 min before loading. Gels were run using a MiniProtean II system (Bio Rad Laboratories) and stained with Coomassie brilliant blue (Sigma Immunochemicals). The molecular weight markers used were Dalton VIIL (Sigma Immunochemicals). Gels were also silver stained using a commercial kit (Gelcode ${ }^{\circledR i l v e r ~ s t a i n ~ k i t ; ~ P i e r c e, ~ R o c k f o r d, ~ I L) ~ t o ~ d e t e c t ~ b o t h ~ t h e ~}$ presence of protein and carbohydrate.

Two-dimensional PAGE. Two-dimensional PAGE gels were run according to the method of O'Farrell (22). Gels were run using a MiniProtean II system and stained with Coomassie blue, with similar molecular weight markers as above. The first dimension, isoelectric focusing, was over the $\mathrm{pI}$ range of 3-10. Second dimension separation was by molecular mass using a $12 \%$ SDS-PAGE gel.

Immunoblotting. Samples separated on one- or two-dimensional 
SDS-PAGE were electroblotted onto Immobilon P polyvinyldifluoride membranes (Millipore Corp., Bedford, MA) overnight (23). Membranes were washed with PBS containing 0.1\% Triton X-100 (Sigma Immunochemicals) (PBS-T) and blocked with PBS-T containing 2\% FCS (blocking buffer) (Sera-Lab). Blocked membranes were then incubated with the test antibody (in blocking buffer) for $1 \mathrm{~h}$ and washed with PBS-T. Bound mouse IgG was detected using peroxidase-labeled goat anti-mouse IgG ( $\gamma$-chain specific) (Sigma Immunochemicals) at 1:1,000 in PBS-T-2\% FCS. After a final wash the blots were developed

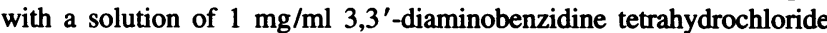
(Sigma Immunochemicals) in $50 \mathrm{mM}$ Tris (Sigma Immunochemicals), $\mathrm{pH} 7.6$, containing $150 \mathrm{mM} \mathrm{NaCl}$ (BDH) and $0.05 \%$ hydrogen peroxide (Sigma Immunochemicals). Each reaction was terminated by extensive rinsing with distilled water.

Protein purification. Crude SAM was fractionated at $4^{\circ} \mathrm{C}$ on a QSepharose anion exchange column $(50 \mathrm{~cm} \times 1.6 \mathrm{~cm})$. The column was equilibrated in $20 \mathrm{mM}$ Tris- $\mathrm{HCl}, \mathrm{pH} 8.5$ (buffer A), and the SAM (generally $100-400 \mathrm{mg}$ ) was loaded on in the same buffer. The column was washed with $500 \mathrm{ml}$ of buffer $A$ and then eluted with a $1,000-\mathrm{ml}$ linear gradient of $0-2 \mathrm{M} \mathrm{NaCl}$ in buffer A. 10-ml fractions were collected, and the absorbance at $280 \mathrm{~nm}$ was monitored. The location of the osteolytic protein was determined by a combination of activity assay, SDS-PAGE, and Western blot analysis. Fractions containing osteolytic activity were dialyzed against deionized water to remove salt and lyophilized. The fraction with the highest specific activity and the least number of protein bands on SDS-PAGE was then further fractionated at room temperature on a second anion exchange column. There was some evidence of proteolytic clipping of the $62-\mathrm{kD}$ protein and so aliquots of this fraction were dissolved at $1 \mathrm{mg} / \mathrm{ml}$ in $20 \mathrm{mM}$ Tris, $\mathrm{pH} 7.2$, containing proteinase inhibitors ( $1 \mathrm{mM}$ PMSF, $1 \mathrm{mM}$ EDTA, and $1 \mathrm{mM}$ benzamidine) (buffer $B$ ) and fractionated on an EconoPak $Q$ column (Bio Rad Laboratories) equilibrated in buffer B. Fractions were eluted by application of a gradient of $0-1.5 \mathrm{M} \mathrm{NaCl}$ in buffer $\mathrm{B}$, and an absorbance profile at $280 \mathrm{~nm}$ was obtained. Fractions were again analyzed for osteolytic activity and Western immunoblotted with mAb P3 to confirm the presence of the immunogenic $62-\mathrm{kD}$ protein. The purity of the fractions was again assessed visually by SDS-PAGE, and $100 \mu \mathrm{g}$ of the cleanest fraction was dialyzed against $50 \mathrm{mM}$ of Tris buffer, $\mathrm{pH}$ 7.6 , containing $10 \mathrm{mM} \mathrm{KCl}$ and $10 \mathrm{mM} \mathrm{MgCl}_{2}$ (buffer C). This sample was run on a 5-ml ATP-Sepharose (Sigma Immunochemicals) column. The column was washed with 10 column volumes of buffer $\mathrm{C}$ and bound protein eluted in 5 column volumes of $5 \mathrm{mM}$ ATP (Sigma Immunochemicals), also in buffer C. Protein was located by SDS-PAGE and visualized using a silver stain kit (Sigma Immunochemicals). Gel filtration was used to determine the molecular mass range of the osteolytic protein isolated by ATP-affinity chromatography. This was done by running the purified protein on a Bio-Sil TSK250 (Bio Rad Laboratories) column in $0.1 \mathrm{M}$ sodium phosphate buffer, $\mathrm{pH} 6.7$, and measuring absorption at $205 / 280 \mathrm{~nm}$.

Immunoaffinity purification. Affinity columns were prepared using $\mathrm{mAb} P 2$, mAb P3, and both P2 and P3 together. Briefly, in each case, $5 \mathrm{mg}$ of antibody was linked to $3.5 \mathrm{ml}$ of swollen cyanogen bromideactivated Sepharose 4B (Sigma Immunochemicals) in bicarbonate buffer at $\mathrm{pH}$ 8.3. After washing, the column was blocked with $1 \mathrm{M}$ ethanolamine at $\mathrm{pH}$ 8.0, washed, and equilibrated in PBS. Crude SAM $(5 \mathrm{mg})$ dissolved at $1 \mathrm{mg} / \mathrm{ml}$ in PBS was loaded on, the column was washed extensively, and bound protein was eluted using $0.1 \mathrm{M}$ glycine $\mathrm{pH}$ 2.5. Eluted fractions of $1 \mathrm{ml}$ were concentrated by using Minicon microconcentrators with cutoff membranes of $10 \mathrm{kD}$ (Amicon, Inc., Beverly, MA), to a volume of $\sim 50 \mu \mathrm{l}$. These were analyzed by SDSPAGE and immunoblotting and tested for osteolytic activity in the calvarial bone resorption assay.

Protein sequencing. Material eluting from the ATP-Sepharose column was run on a 10\% SDS-PAGE gel according to the method of Laemmli (21) and electroblotted onto ProBlott membrane (Applied Biosystems Inc., Foster City, CA). The band of interest, at $62 \mathrm{kD}$, was excised and run on an ABI 470A protein sequencer (Applied Biosystems
Table I. Composition of the SAM from the Various Bacteria Studied

\begin{tabular}{|c|c|c|c|c|}
\hline \multirow[b]{2}{*}{ Bacterium } & \multicolumn{4}{|c|}{ Composition (\%) } \\
\hline & Protein & Carbohydrate & Nucleic acid & LPS \\
\hline & & & & $I U / n g$ \\
\hline A. actinomycetemcomitans & $60-70^{*}$ & $12-15^{*}$ & $8-10^{*}$ & $9 \times 10^{-3 t}$ \\
\hline E. corrodens & 60 & 6 & 14 & $6 \times 10^{-4}$ \\
\hline P. gingivalis & $40-50 *$ & 18 & 3 & $2 \times 10^{-5}$ \\
\hline S. aureus & $35-45^{*}$ & 5 & 5 & + \\
\hline
\end{tabular}

* Range of estimates from 5 to 15 different preparations of SAM from each organism. ${ }^{\ddagger} 1 \mu \mathrm{g} E$. coli LPS contains 7,000 IU. +, Generally below limit of assay detection.

Inc.) for 40 cycles using an ABI "Blott" cartridge and an optimized program for electroblotted samples. Data were collected and analyzed using Waters Expert-Ease software (Millipore Corp.).

Heat shock proteins. Additional purified GroEL-like (chaperonin $60)$ proteins were obtained and tested for osteolytic activity in the calvarial bone resorption assay. GroEL-like proteins from Mycobacterium leprae and Mycobacterium tuberculosis were supplied by the World Health Organization antigen bank (Braunschweig, Germany). E. coli GroEL, was obtained commercially (Boehringer Mannheim, Mannheim, Germany). These proteins were separated by SDS-PAGE, Western blotted, and probed with antibody P3 to determine if they contained a cross-reactive epitope. Each specimen was also tested for osteolytic activity. Before testing, each sample was passed through a DeToxi-Gel column (Pierce), as per manufacturer's directions, to remove any LPS contamination. Removal of LPS was checked by running material before and after the DeToxi-Gel column on SDS-PAGE and silver staining the gels. Samples of the various LPS-free GroEL homologues were then diluted and tested over a limited concentration range to determine their potency and efficacy in the murine calvarial bone resorption assay.

\section{Results}

Composition of the SAM. The composition of the SAM from the various bacteria is shown in Table I. The SAM from $A$. actinomycetemcomitans when analyzed by two-dimensional PAGE demonstrated the presence of $\sim 50$ Coomassie bluestaining spots in the pI range of 3-10 and of molecular masses ranging from $<14$ to $>66 \mathrm{kD}$ (Fig. 1).

Activity of SAM in the murine calvarial bone resorption assay. The SAM extracted from A. actinomycetemcomitans showed a consistent profile of activity (Fig. 2) with calcium release increasing linearly over the concentration range from 0.01 to $10 \mu \mathrm{g} / \mathrm{ml}$. With some batches of SAM, significant bone resorbing activity was found at a concentration of $1 \mathrm{ng} / \mathrm{ml}$.

$Q$-Sepharose anion-exchange chromatography. Osteolytic activity was seen to elute at a salt concentration in the range of $0.9-0.92 \mathrm{M}$ in four consecutive fractions (28-31) (Fig. $3)$. These bioactive fractions represented $\sim 3 \%$ of the protein applied to the column. Active fractions contained a prominent $62-\mathrm{kD}$ protein band on SDS-PAGE stained with Coomassie blue.

Neutralization of bone resorption by mAbs to A. actinomycetemcomitans. mAbs raised to whole A. actinomycetemcomitans were tested in the bone resorption assay to determine if they would have any effect on bone breakdown induced by 


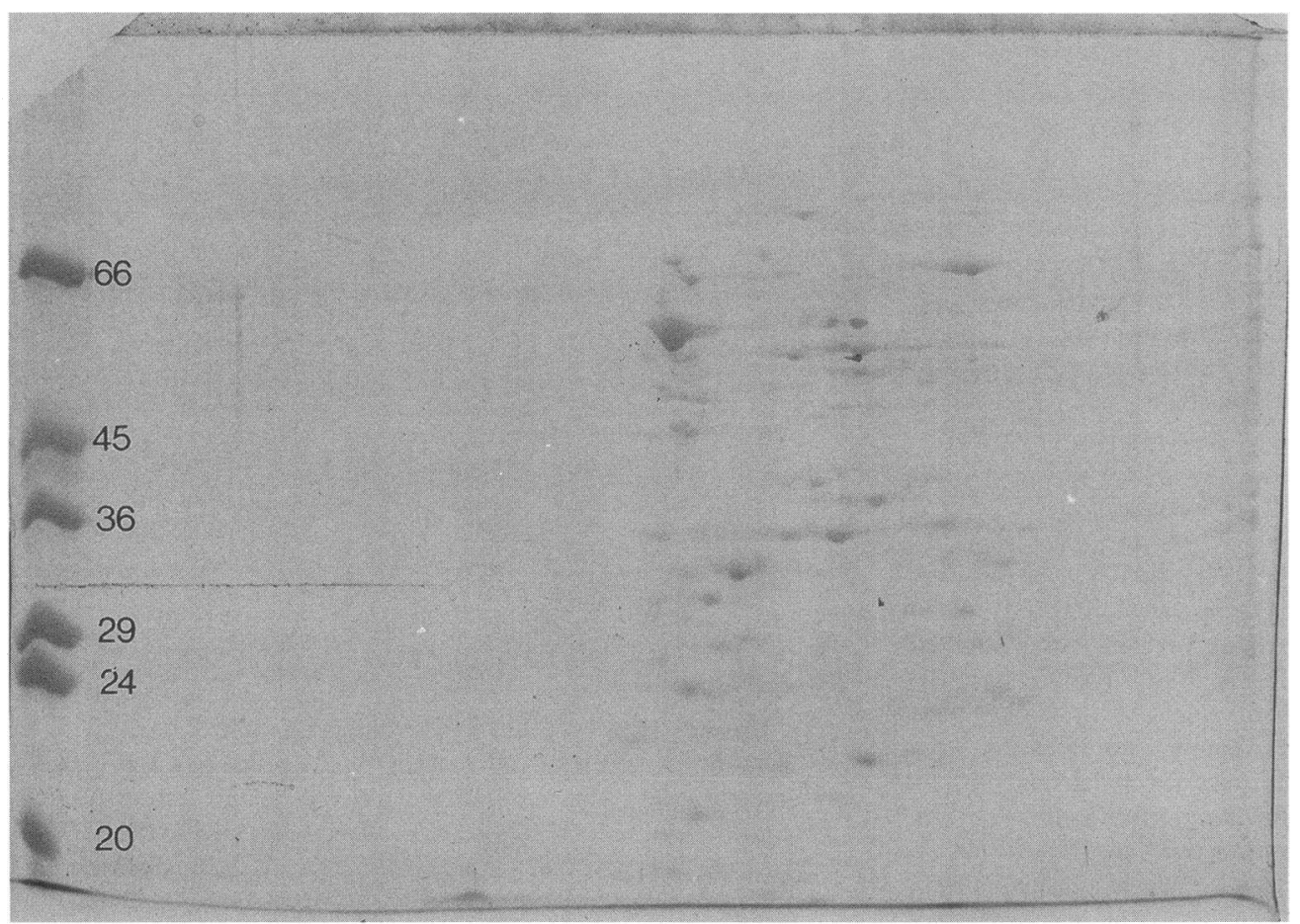

Figure 1. Determination of the protein composition of the SAM of $A$. actinomycetemcomitans by two-dimensional SDS-PAGE. Proteins have been separated in one dimension on the basis of their isoelectric point and in the other dimension on the basis of their molecular mass. The gel was stained with Coomassie blue to disclose proteins. The molecular mass markers are displayed on the left-hand side.
SAM. The inclusion of nonspecific mouse IgG or mAbs P1 or A5 had no noticeable effect on the bone resorbing activity of the SAM, even at concentrations as high as $100 \mu \mathrm{g} / \mathrm{ml}$. In contrast, mAbs P2 and P3 inhibited calcium release. P3 completely inhibited the bone resorbing activity of the SAM when added at a concentration of $7 \mu \mathrm{g} / \mathrm{ml}$ (Fig. 4), whereas P2 only attained comparable inhibition at a concentration of $100 \mu \mathrm{g} / \mathrm{ml}$. The antibodies had no effect on the bone resorption induced by the osteolytic agonist $\mathrm{PGE}_{2}$. To confirm that antibody-mediated inhibition of bone resorption was associated with binding to components of the SAM, this material was incubated with antibody $\mathrm{P} 3$, and the antibody-antigen complexes produced were immunoprecipitated with heat-killed/formalin-fixed $S$. aureus (Sigma Immunochemicals). It was clear (Fig. 5) that immunoprecipitation with antibody $\mathrm{P} 3$, the most potent neutralizing

Calcium Release (mg/d)

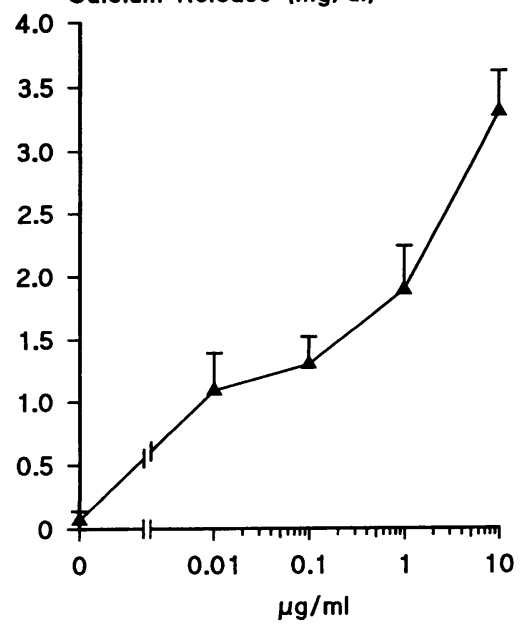

Figure 2. Dose response of the stimulation of calvarial bone resorption induced by the SAM from A. actinomycetemcomitans. Bone breakdown is measured as the release of calcium from the bone. Each point represents the mean and standard deviation of five separate cultures. The concentrations of SAM used ranged from $10 \mathrm{ng} /$ $\mathrm{ml}$ to $10 \mu \mathrm{g} / \mathrm{ml}$. antibody, reduced the osteolytic activity of A. actinomycetemcomitans SAM to background levels. This was seen to be the case even when the depleted fraction was added at a concentration of $10 \mu \mathrm{g} / \mathrm{ml}$. Controls in which SAM was incubated with a nonspecific antibody, or with $S$. aureus alone, retained activity equal to the untreated SAM.

Specificity of $m A$ bs $P 2$ and $P 3$ assessed by Western blotting. Using two-dimensional SDS-PAGE of A. actinomycetemcomitans SAM, many proteins or protein subunits were separated (Fig. $6 a$ ). Immunoblotting these preparations using mAbs P2, P3 (Fig. $6 b$ ), or a combination of both showed that both mAbs recognized the same protein, which had a molecular mass of $62 \mathrm{kD}$.

Affinity purification of the $62-k D$ osteolytic protein. Affinity columns containing mAbs $\mathrm{P} 2, \mathrm{P} 3$, or a combination of both antibodies, linked to Sepharose 4B, were used to try and achieve a one-pass purification of the active protein. Both antibodies appeared to bind very weakly to the $62-\mathrm{kD}$ protein and it was only possible to isolate small quantities of this protein by this technique. The isolated protein was active in the bone resorption assay and reacted with antibodies P2 and P3 in Western blots.

In a prior study a small quantity of the $62-\mathrm{kD}$ protein which bound to antibody P3 was used for sequencing. This suggested homology with the $E$. coli heat shock protein GroEL (24). It had been demonstrated that purification of this molecular chaperone can be achieved simply by affinity purification on an ATP column (25). When the anion exchange chromatographypurified fractions containing bone-resorbing activity were passed through an ATP-Sepharose column and the column was washed and then eluted with either ATP or magnesium-free buffer, a single protein was eluted as seen on a silver-stained SDS-PAGE gel (Fig. 7). This protein was active in the bone resorption assay and, when Western blotted, was recognized by mAbs P2 and P3 but not by mAbs P1 or A5 or by normal 


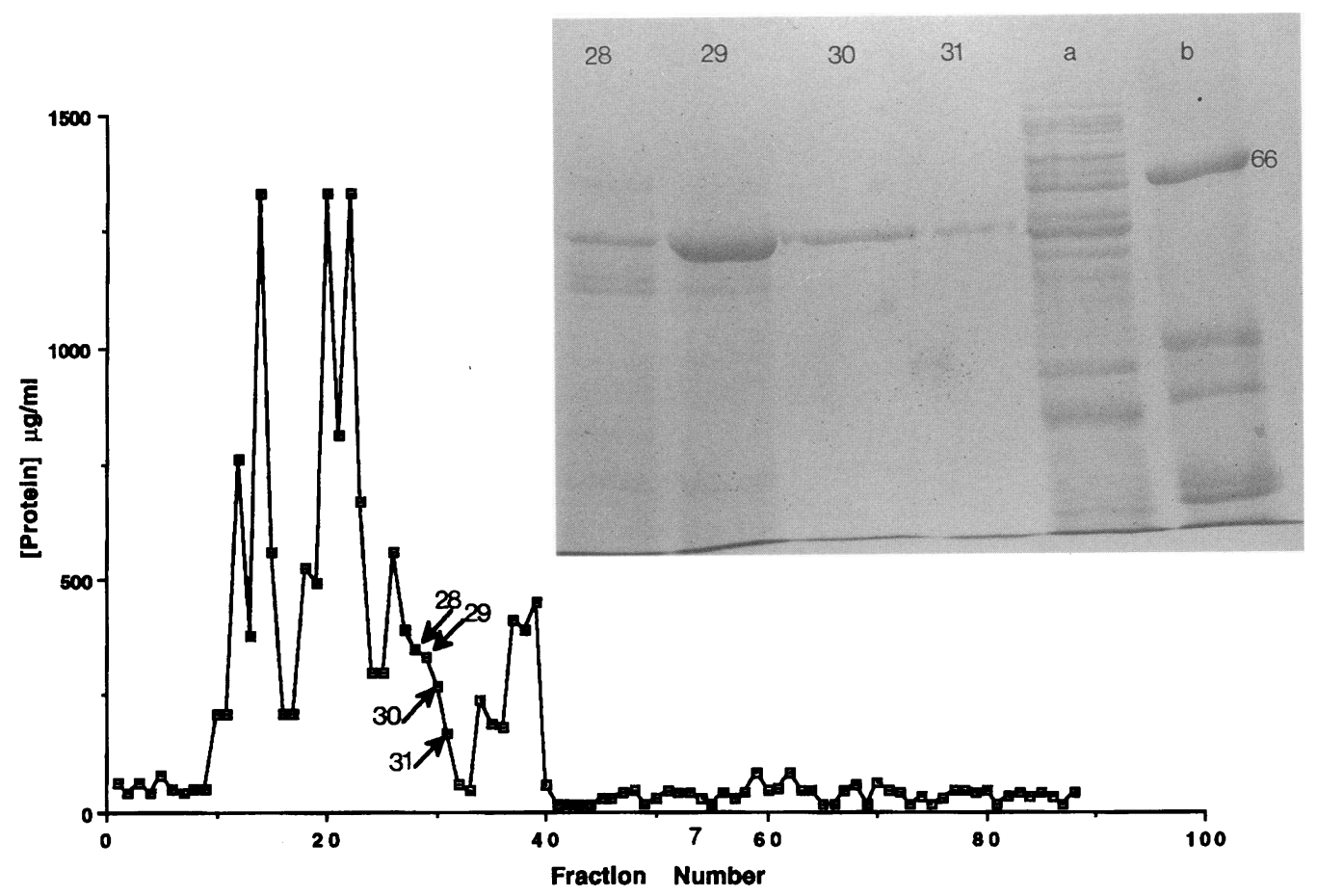

Figure 3. Elution profile of the SAM on Q-Sepharose anion exchange chromatography. Each fraction was assessed for protein content and for bone-resorbing activity in the murine calvarial bone resorption assay. The protein content of each fraction is shown and the fractions with osteolytic activity are arrowed. The SDS-PAGE profiles of bioactive fractions are shown in the inset. Lane $a$ is the starting material and lane $b$ contains the molecular mass markers with the 66-kD marker highlighted. Osteolytic activity is associated with the presence of a $62-\mathrm{kD}$ protein in the fractions.

mouse IgG. When the protein isolated from the ATP column was fractionated by gel filtration on a TSK250 column, which has a molecular exclusion limit of $300 \mathrm{kD}$, osteolytic activity eluted in the void volumn fraction.

$\mathrm{NH}_{2}$-terminal sequencing. $\mathrm{NH}_{2}$-terminal sequencing of the $62-\mathrm{kD}$ protein eluted from the ATP-affinity column produced a continuous sequence of 38 residues. Comparisons using the National Centre for Biotechnology Information Blast network service showed this sequence to have $100 \%$ homology to the GroEL protein of $E$. coli over the first 17 residues and to differ at only 2 of the 38 residues sequenced. The homology to other GroEl-like proteins such as those from mycobacterial species or the human P1 protein was in the order of $60-70 \%$ (Table II).

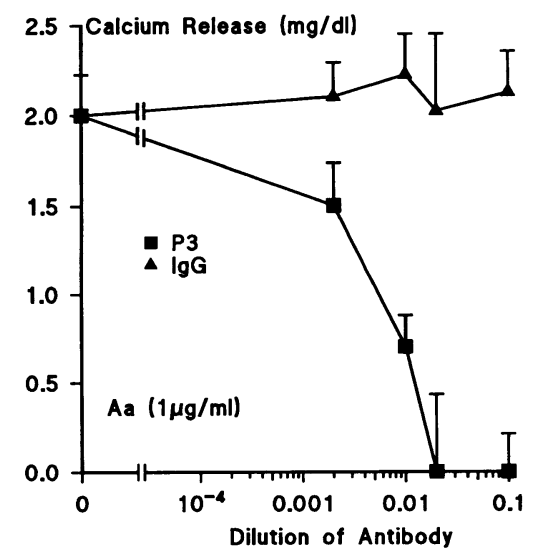

Figure 4. Dose-dependent inhibition of the bone resorption (measured as calcium release) induced by $1 \mu \mathrm{g} / \mathrm{ml}$ SAM from A. actinomycetemcomitans by addition of $\mathrm{mAb} P 3$ to the bone explants. The lack of effect of equivalent concentrations of mouse IgG is also seen. Results are expressed as the mean and standard deviation of five replicate cultures.
Cell surface expression of GroEL. Saline extracts of bacteria whose SAMs are known to stimulate bone resorption, $E$. corrodens, $P$. gingivalis, and $S$. aureus, and a crude extract of $E$. coli, were Western blotted and reacted with antibody P3. Only

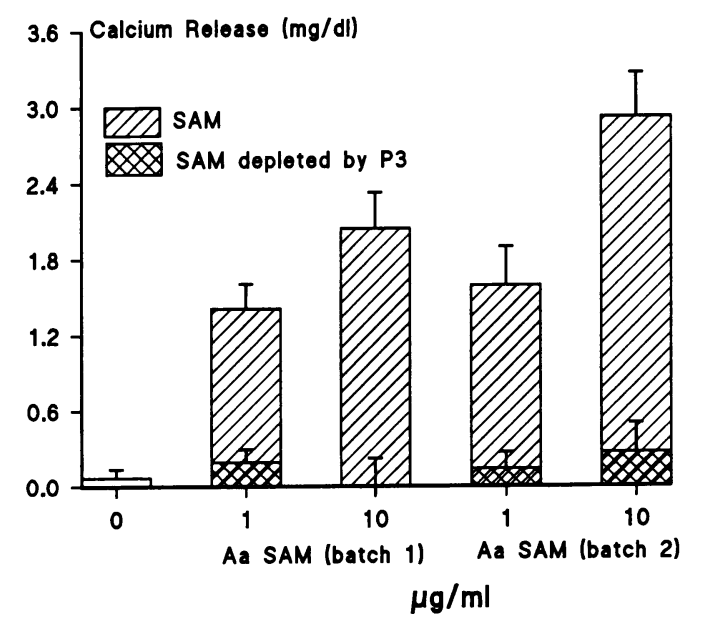

Figure 5. Effect of depletion of the SAM, by immunoabsorption with $\mathrm{mAb} \mathrm{P} 3$, on the stimulation of murine calvarial bone resorption. Cultures were exposed to 1 or $10 \mu \mathrm{g} / \mathrm{ml} \mathrm{SAM}$ which had either been depleted with $\mathrm{mAb}$ P3 or sham-depleted by incubation with heat-killed $S$. aureus (Cowan strain) in the absence of P3. Results are expressed as the mean and standard deviation of five replicate cultures. Two individual preparations (batches) of the SAM have been tested to show the batch-to-batch reproducibility. The column denoted 0 shows the amount of calcium released by unstimulated calvaria. 

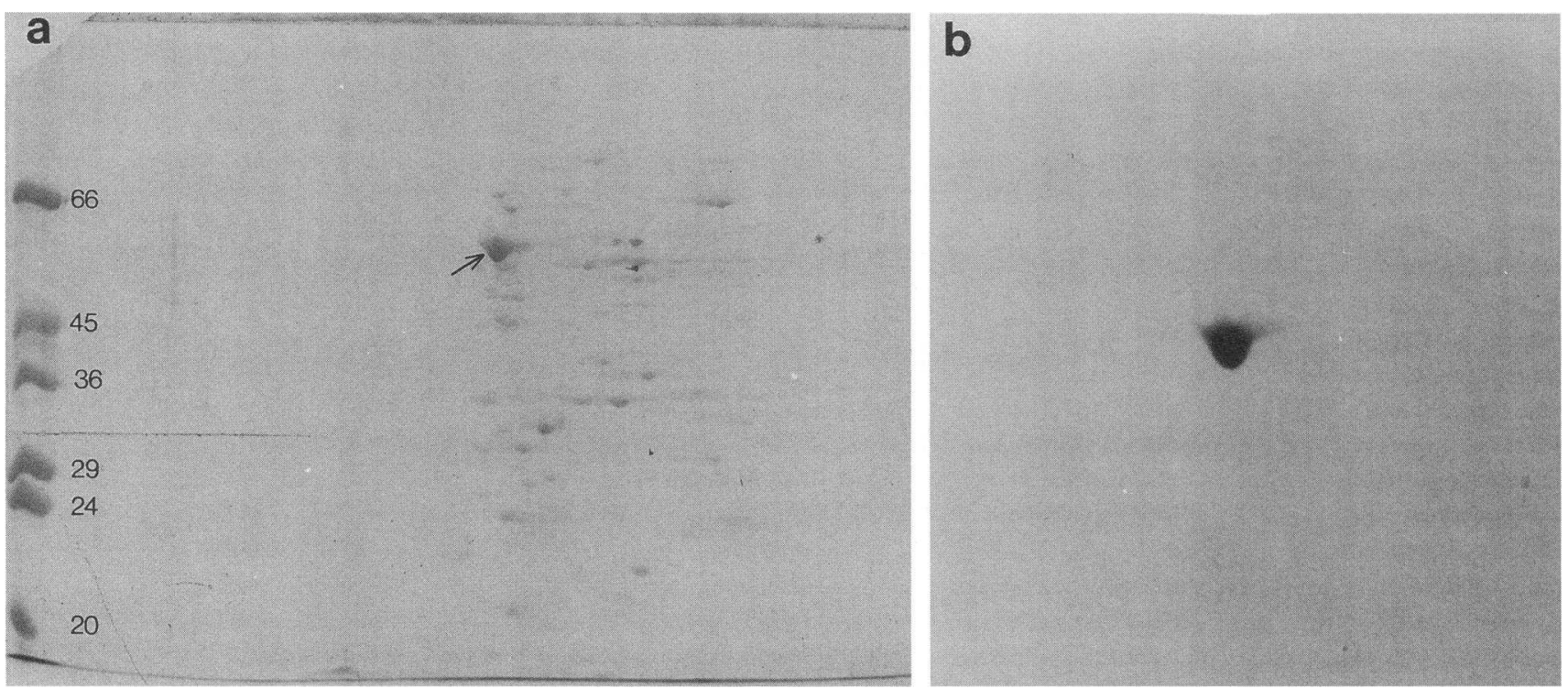

Figure 6. Western immunoblotting of A. actinomycetemcomitans SAM separated by two-dimensional SDS-PAGE with mAb P3. (a) Coomassie blue-stained two-dimensional gel with a prominent protein spot of $62 \mathrm{kD}$ arrowed; $(b)$ gel immunoblotted with antibody P3 to demonstrate that this antibody only binds to this $62-\mathrm{kD}$ protein.

the SAM from A. actinomycetemcomitans showed the presence of the specific $62-\mathrm{kD}$ antigen in this surface-associated fraction (Fig. 8).

Bone-resorbing activity of other bacterial GroEL-like proteins. The GroEL-like proteins from M. leprae and $M$. tuberculosis (i.e., hsp 60) and E. coli GroEL, when Western immunoblotted, reacted with antibody P3 (Fig. 8), showing that these proteins shared the epitope which $\mathrm{P} 3$ recognized and which was associated with the inhibition of the bone-resorbing activity. However, when these various purified proteins were tested for activity in the calvarial bone resorption assay only that from $E$. coli had the capacity to stimulate resorption after removal of associated LPS (Fig. 9). LPS removal was confirmed by run-

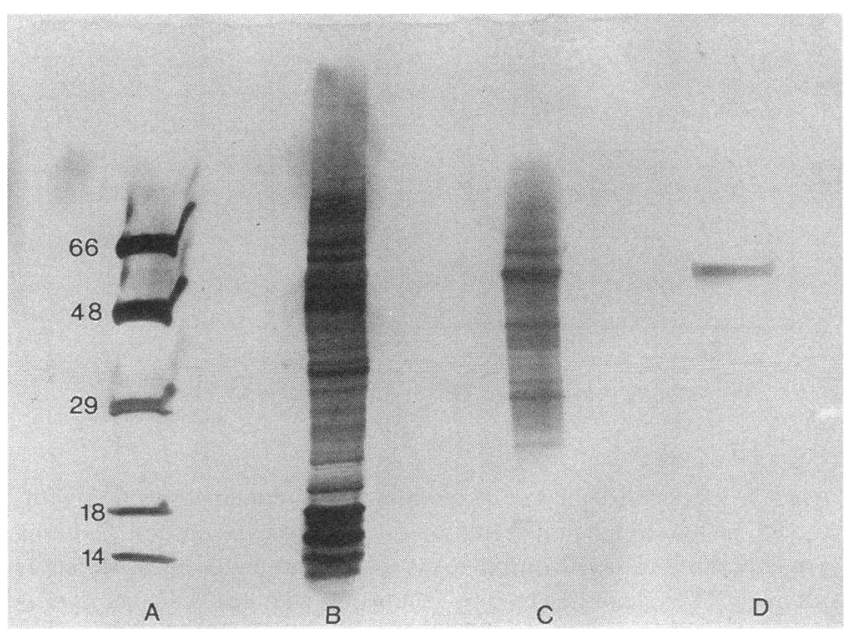

Figure 7. Affinity purification of the 62-kD osteolytic protein on ATPSepharose. $(A)$ Molecular weight standards; $(B)$ starting material; $(C)$ protein eluted from Q-Sepharose column; $(D)$ material eluted from ATP-Sepharose column. The SDS-PAGE gel was stained with silver. ning the GroEL preparations on overloaded SDS-PAGE and silver staining the gels to look for the characteristic LPS ladder pattern.

Role of LPS. The possibility that the osteolytic activity of the SAM was due to either LPS contamination or synergy between LPS and the GroEL homologue was addressed. The starting material (SAM) had low levels of endotoxin, and silverstained SDS-PAGE gels overloaded with the purified GroEL homologue failed to show the ladder pattern characteristic of LPS (Fig. 10). Polymyxin B inhibited the bone-resorbing activity of the LPS from A. actinomycetemcomitans but failed to inhibit the osteolytic activity of the purified GroEL homologue from this bacterium (Table III). In contrast, antibody P3 inhibited the osteolytic activity of the GroEL homologue but failed to inhibit the activity of the LPS from A. actinomycetemcomitans (Table III). In addition, the osteolytic activity of the GroEL

Table II. $\mathrm{NH}_{2}$-terminal Amino Acid Sequences of GroEL and GroEL-like Proteins

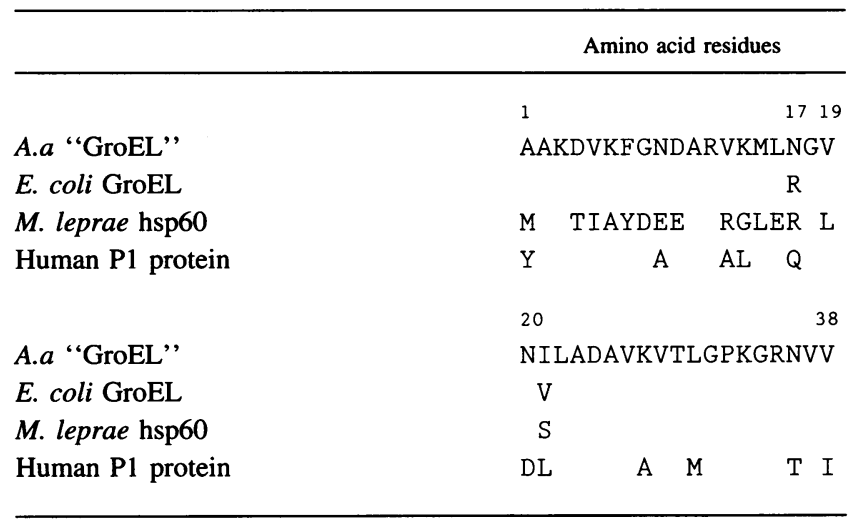

A.a, A. actinomycetemcomitans. 


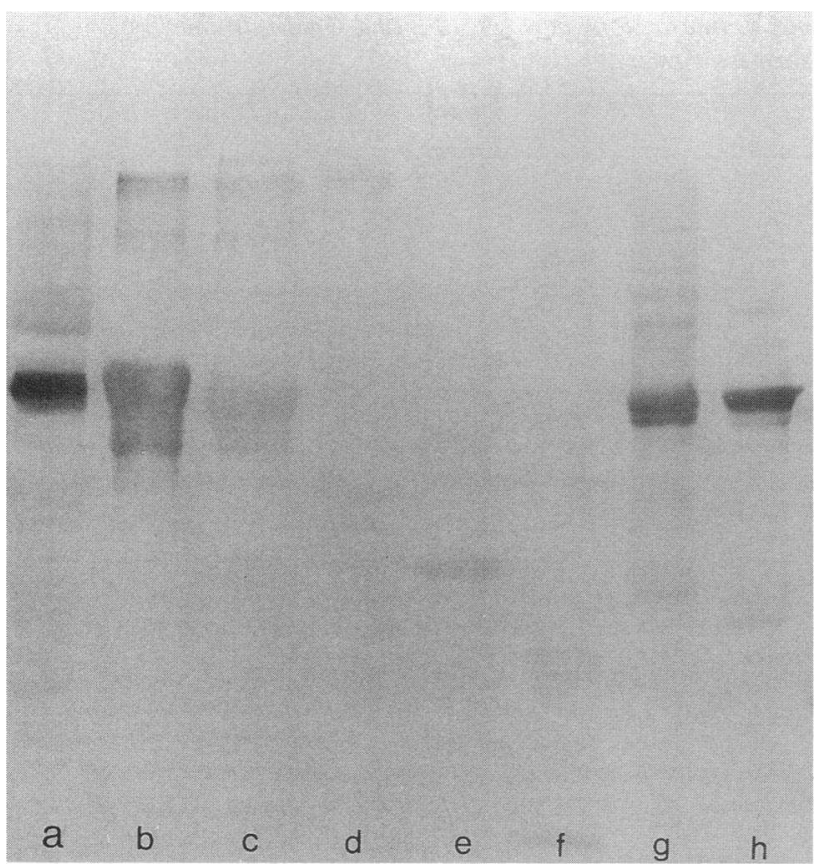

Figure 8. Western immunoblotting of bacterial components with antibody P3: $(a)$ a crude lysate of $E$. coli; $(b) M$. tuberculosis hsp65; (c) M. leprae hsp65; (d) SAM from S. aureus; (e) SAM from P. gingivalis; $(f)$ SAM from $E$. corrodens; $(g)>30-\mathrm{kD}$ fraction of the SAM from A. actinomycetemcomitans; $(h)$ crude SAM from A. actinomycetemcomitans.

homologue was sensitive both to heating and to trypsin (Table III). SAM and LPS from A. actinomycetemcomitans were also tested in the calvarial assay using $\mathrm{C} 3 \mathrm{H} / \mathrm{HeJ}$ mice which are unresponsive to LPS. The combined results from two separate experiments are shown in Table IV, which shows clearly that while the calvarial bone is responsive to the SAM it is unresponsive to the LPS from this organism.

To ascertain if LPS and GroEL interacted in a synergistic manner in the bone resorption assay, suboptimal concentrations of both components were added singly or together to calvaria and the amount of bone resorption was determined $48 \mathrm{~h}$ later. No evidence of synergistic interactions was noted, indeed

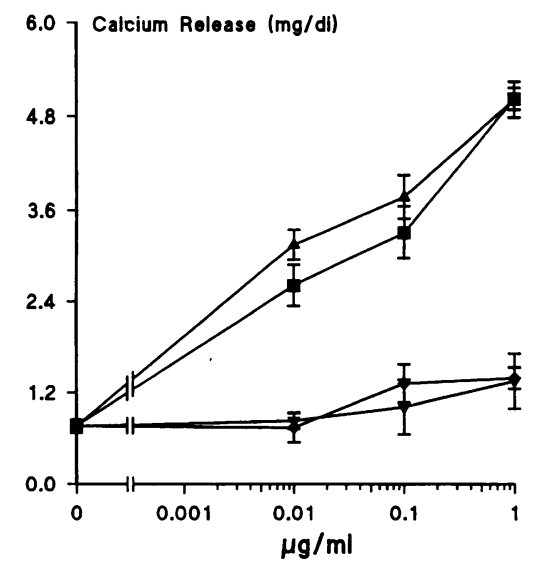

Figure 9. Stimulation of calvarial bone resorption by increasing concentrations of SAM from A. actinomycetemcomitans

$(\boldsymbol{\square})$, purified $E$. coli GroEL ( $\Lambda$ ), or the GroEL-like proteins from M. tuberculosis ( $\bullet$ ) or $M$. leprae ( $\mathbf{\nabla})$. Results are expressed as the mean and standard deviation of five replicate cultures. The concentration range is from $10 \mathrm{ng} / \mathrm{ml}$ to $1 \mu \mathrm{g} / \mathrm{ml}$.
$66 \mathrm{kD}$

48.5kD

$29 k D$

$18.4 \mathrm{kD}$

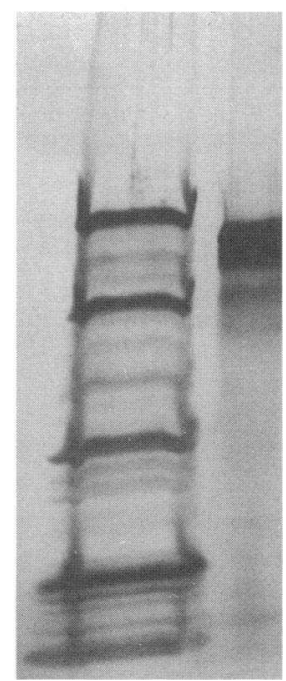

Figure 10. Silver-stained SDSPAGE gel overloaded with the purified GroEL homologue $(B)$ showing lack of a ladder pattern indicative of LPS contamination. The molecular mass markers are shown $(A)$.

the two components failed to produce an additive response (Table V).

\section{Discussion}

LJP is characterized by the severe and rapid loss of alveolar bone on the approximal surfaces of first molar and/or incisor teeth and is generally associated with the presence of the Gramnegative bacterium $A$. actinomycetemcomitans $(1,2)$. Work from the Eastman Dental Institute has established that gentle saline extraction of this organism releases a proteinaceous fraction that is assumed to be associated with the external surface of the outer membrane. Electron microscopic examination of bacteria after extraction failed to show evidence of cell disruption $(5,6)$. The finding of extremely low LPS levels in the SAM is additional evidence of the lack of postextraction cell lysis. Of course it cannot be conclusively proven that all proteins in the SAM come from the cell surface and the term is therefore

Table III. Effect of Various Treatments on the Capacity of the A. actinomycetemcomitans GroEL Homologue or LPS to Stimulate Calvarial Bone Resorption

\begin{tabular}{lc}
\multicolumn{1}{c}{ Treatment } & Percentage of inhibition \\
\hline GroEI homologue + & 3 \\
Polymyxin B & 62 \\
$100^{\circ} \mathrm{C}$ for $30 \mathrm{~min}$ & 79 \\
Trypsin for 60 min & 81 \\
mAb P3 & \\
A. actinomycetemcomitans LPS + & 79 \\
Polymyxin B & 2 \\
100 $\mathrm{C}$ for 30 min & 3 \\
Trypsin for 60 min & 6 \\
mAb p3 & \\
\hline
\end{tabular}

The details of the methodology used are given in Methods. 
Table IV. Bone Resorption of $\mathrm{C} 3 \mathrm{H} / \mathrm{HeJ}$ Strain Calvaria in Response to SAM or LPS from A. actinomycetemcomitans

\begin{tabular}{lc}
\hline \multicolumn{1}{c}{ Stimulant } & Calcium release \\
\hline & $m g / d l$ \\
A.a SAM $(\mu \mathrm{g} / \mathrm{ml})$ & \\
10 & $3.6 \pm 0.7$ \\
1 & $1.6 \pm 0.3$ \\
0.1 & $0.8 \pm 0.2$ \\
0.01 & $0.3 \pm 0.2$ \\
A. $\mathrm{LPS}(\mu \mathrm{g} / \mathrm{ml})$ & \\
10 & $0.4 \pm 0.1$ \\
1 & $0.2 \pm 0.1$ \\
0.1 & $0.2 \pm 0.2$ \\
0.01 & $0.3 \pm 0.1$ \\
Media control (no stimulators) & $0.2 \pm 0.1$ \\
rIL-1 $\alpha(10 \mathrm{ng} / \mathrm{ml})$ & $4.2 \pm 0.9$
\end{tabular}

Results are expressed as the mean and standard deviation of two separate experiments in which each concentration of agonist was tested in five replicate cultures. A.a, A. actinomycetemcomitans.

an operational one for material eluted when bacteria are stirred in isotonic saline. This surface-associated material has a range of biological actions, the most prominent being its ability to stimulate the breakdown of murine calvarial bone, an assay used to detect the activity of osteolytic agents. Significant stimulation of bone resorption can be induced with $1-10 \mathrm{ng} / \mathrm{ml}$ of this crude fraction. An interesting finding in contemporaneous experiments is that, in contrast to the SAM from $P$. gingivalis (26), E. corrodens (6), or $S$. aureus (27), the bone-resorbing activity of the SAM from A. actinomycetemcomitans cannot be inhibited by nonsteroidal antiinflammatory drugs or by inhibiting the actions of the potent osteolytic cytokines IL-1 or TNF $\alpha$ (6). Thus the active component in the SAM appears not to be able to induce key mediators normally associated with the induction of calvarial bone resorption (4). This suggests that the active moiety in the SAM is interacting directly with bone cells to induce resorption. Our preliminary studies suggest that the active protein can directly stimulate the recruitment of the primary bone-resorbing cell (the osteoclast), but we cannot rule out a direct effect on osteoblasts (6).

A substantial degree of purification of the osteolytic activity of the SAM was achieved by anion exchange chromatography and activity appeared to be associated with a $62-\mathrm{kD}$ protein. Further purification, using anion exchange chromatography, failed to isolate the active protein to homogeneity. We had shown previously that sera from a proportion of patients with LJP could block the osteolytic activity of the SAM from $A$. actinomycetemcomitans (28) and we had begun a program to develop and test monoclonal antibodies to the SAM to both aid purification and to provide probes for studies of the biological activity of this material. Two antibodies were found that inhibited the SAM-induced resorption of bone, and one of these antibodies, P3, was able to remove the bone-resorbing activity by immunoabsorption. In contrast, normal mouse serum or mAbs P1 and A5 (which are of the same IgG1 subclass as mAbs $\mathrm{P} 2$ and $\mathrm{P} 3$ ) had no inhibitory activity. When antibodies $\mathrm{P} 2$ and $\mathrm{P} 3$ were used in Western blots to identify the antigen,
Table V. Interactions between LPS and GroEL in the Bone Resorption Assay

\begin{tabular}{lc}
\hline \multicolumn{1}{c}{ Combination } & Calcium release \\
\hline & $m g / d l$ \\
Control & $0.1 \pm 0.1$ \\
PGE $($ maximal release) & $4.1 \pm 0.7$ \\
A.a GroEL $(100 \mathrm{ng} / \mathrm{ml})$ & $1.3 \pm 0.3$ \\
A.a LPS $(1 \mu \mathrm{g} / \mathrm{ml})$ & $1.6 \pm 0.2$ \\
GroEL + LPS & $1.8 \pm 0.2$ \\
E. coli GroEL $(100 \mathrm{ng} / \mathrm{ml})$ & $0.9 \pm 0.2$ \\
E. coli LPS $(10 \mathrm{ng} / \mathrm{ml})$ & $2.1 \pm 0.5$ \\
GroEL + LPS & $2.2 \pm 0.1$
\end{tabular}

Results show the release of calcium from murine calvaria exposed to either GroEL or LPS or to a combination of both agents. The control value is the calcium release from unstimulated bone. Maximal stimulation is induced by adding $1 \mu \mathrm{g} / \mathrm{ml} \mathrm{PGE} 2$. A.a, A. actinomycetemcomitans.

they both recognized a $62-\mathrm{kD}$ protein. Preliminary studies had suggested that these antibodies recognized a protein that had some homology with the $E$. coli molecular chaperone or heat shock protein GroEL (24). GroEL has ATP binding activity, and this activity provides a convenient method for its purification (25). On the basis of this information, we then used a previously described ATP-Sepharose chromatography technique $(25)$ to isolate the $62-\mathrm{kD}$ protein. The use of the ATPcolumn resulted in the isolation of a homogenous $62-\mathrm{kD}$ protein, as assessed by silver-stained SDS-PAGE, with total recovery of bone-resorbing activity. $\mathrm{NH}_{2}$-terminal sequencing showed that of the first 38 residues identified, 36 were identical to that of $E$. coli GroEL, and one was a conservative substitution. The homology to the GroEL-like proteins of mycobacterial species was in the region of $60-70 \%$ with similar homology to the mitochondrial P1 GroEL homologue (Table II). Electron microscopic examination of negatively stained preparations of this A. actinomycetemcomitans GroEL-like protein showed the characteristic double ring structure with sevenfold symmetry (25) (our unpublished data).

We conclude that the surface-associated material from the oral bacterium A. actinomycetemcomitans contains a homologue of the E. coli molecular chaperone or heat shock protein, GroEL, and that this protein is responsible for the potent bone-resorbing activity of this fraction. When the purified GroEL homologue was run through a TSK250 gel filtration column that had a molecular exclusion limit of $300 \mathrm{kD}$, the boneresorbing activity appeared in the void volume, implying that the GroEL multimer is the active moiety. The finding that a homologue of GroEL is osteolytic is surprising, not the least because the SAM fraction is assumed to contain only material associated with the bacterial outer surface and GroEL is generally assumed to be an intracellular protein. Even if this protein is not associated with the cell surface, it is rapidly removed from the cells by gentle washing in isotonic saline. We have shown that the SAM fraction from a number of oral bacteria, such as $P$. gingivalis (26), $E$. corrodens (6), and $S$. aureus (27), expresses potent osteolytic activity. This raised the possibility that the activity of these SAMs may have been due to the presence of GroEL-like proteins. However, no antigen cross-reactive with mAbs P2 or P3 
was found in the SAM fractions isolated from these bacteria and their osteolytic activity could not be blocked by P3 (results not shown). Thus A. actinomycetemcomitans, at least in culture, appears to be unusual in that it may express GroEL on its surface although we have no evidence that this is the case in vivo. It should be noted that this is not a unique finding as surface expression of a GroEL homologue in Helicobacter pylori was also suggested by the fact that the protein could be removed by vortexing in distilled water and was capable of being labeled by surface-labeling techniques $(29,30)$. However, a direct pathogenic action of this $H$. pylori GroEL homologue has not been claimed. Heat shock proteins are major antigens in a variety of infectious and autoimmune diseases $(31,32)$. We have also established that: $(a)$ the $62-\mathrm{kD}$ protein of A. actinomycetemcomitans is an immunodominant antigen as assessed by Western blotting with sera from patients with LJP; and $(b)$ that a proportion of patients with strong circulating antibody responses to the SAM has the capacity to neutralize its osteolytic activity and thus almost certainly contains antibodies directed against GroEL with similar specificities to P2 and P3 (28).

The GroEL homologue is a potent stimulator of bone resorption with some preparations of SAM demonstrating bone-resorbing activity at a concentration of $1 \mathrm{ng} / \mathrm{ml}$. We have now established that the active protein has a molecular mass of 62 $\mathrm{kD}$ and that it represents $\sim 2 \%$ of the dry mass of the SAM. Thus, this protein is capable of demonstrating bone-resorbing activity at a molar concentration of $\sim 1 \mathrm{pM}$, a similar potency to that of the most active bone-stimulating agonist, IL-1 (33).

That the osteolytic activity was due to contaminating LPS, or to some interaction between LPS and the GroEL homologue, was a possibility that required investigation. The LPS content of the SAM and the GroEL has been determined by the Limulus assay and was low. One of the authors (T. Nishihara) has shown that the LPS from $A$. actinomycetemcomitans has comparable activity to that of $E$. coli LPS in the Limulus assay (34). A. actinomycetemcomitans LPS produces a classic ladder pattern on silver-stained SDS-PAGE gels (34). No such ladder pattern was seen on an overloaded silver-stained SDS-PAGE gel of the purified GroEL homologue. Thus, it would appear that LPS contamination is minimal. It was further shown that while the osteolytic activity of the LPS from A. actinomycetemcomitans could be inhibited by polymyxin $B$, that of the SAM or the purified GroEL homologue was unaffected. In contrast, the neutralizing mAb P3 had no inhibitory effect on the bone-resorbing activity of LPS. It was also demonstrated that calvaria from the LPS-unresponsive mouse strain $\mathrm{C} 3 \mathrm{H} / \mathrm{HeJ}$ would resorb in response to the SAM but not when stimulated with the LPS from A. actinomycetemcomitans, confirming that the osteolytic activity in the SAM was not due to LPS contamination. The biological effects of LPS are not sensitive to heat or trypsin, yet the bone-resorbing activity of the SAM and the GroEL was significantly inhibited by short-term heating or exposure to trypsin. These findings refute the hypothesis that the boneresorbing activity is due to LPS. It was possible that residual LPS in the SAM could form a complex with the GroEL homologue that demonstrated the property of synergism. This possibility was tested by adding suboptimal concentrations of LPS or GroEL to bone. However, this showed no evidence of synergy and indeed suggested that both components could possibly interfere with each other in inducing bone resorption. Thus, we conclude that the osteolytic effects of the SAM are due to the activity of a GroEL-like chaperone protein and that LPS plays no part in the activity.

We have examined the bone-resorbing activity of GroEL from $E$. coli and GroEL homologues from $M$. tuberculosis and $M$. leprae and have found that the former does indeed demonstrate osteolytic activity but that the latter two (once the LPS has been removed) have little bone-resorbing activity. This difference in osteolytic activity may be due to structural differences in these GroEL proteins, and further studies are underway to clone and sequence the GroEL homologue from A. actinomycetemcomitans.

We therefore speculate that the GroEL homologue of $A$. actinomycetemcomitans functions both as a bone-resorbing virulence factor and as an immunogen. In a proportion of patients, the immune response to this protein is neutralizing, and we are currently setting up studies to determine if this neutralizing immune response plays any role in the clinical course of the bone destruction.

\section{Acknowledgments}

We are grateful to the Medical Research Council for funding P. White, Action Research and the Jules Thorn Trust for funding K. Reddi, and the Arthritis and Rheumatism Research Council for funding S. P. Nair.

\section{References}

1. Slots, J., and R. J. Genco. 1984. Black pigmented bacteroides species, Capnocytophaga species and Actinobacillus actinomycetemcomitans in human periodontal disease: virulence factors in colonization, survival and tissue destruction. J. Dent. Res. 63:412-421.

2. Zambon, J. J. 1985. Actinobacillus actinomycetemcomitans in human periodontal disease. J. Clin. Periodontol. 12:1-20.

3. Liakoni, H., P. Barber, and H. N. Newman. 1987. Bacterial penetration of pocket soft tissues in chronic adult and juvenile periodontitis cases. An ultrastructural study. J. Clin. Periodontol. 14:22-28.

4. Tatakis, D. N. 1993. Interleukin 1 and bone metabolism: a review. J. Periodontol. 62:416-431.

5. Wilson, M., S. Kamin, and W. Harvey. 1985. Bone resorbing activity of purified capsular material from Actinobacillus actinomycetemcomitans. J. Periodontal. Res. 20:484-491.

6. Meghii, S., M. Wilson, P. Barber, and B. Henderson. 1994. Bone resorbing activity of surface-associated material from Actinobacillus actinomycetemcomitans and Eikenella corrodens. J. Med. Microbiol. 41:197-203.

7. Barber, P. M., M. Wilson, S. J. Challacombe, and H. N. Newman. 1992. Localization of surface-associated material (SAM) from Porphyromonas gingivalis on extracted periodontitis affected teeth. J. Dent. Res. 71:552.

8. Meghji, S., M. Wilson, B. Henderson, and D. Kinane 1992. Anti-proliferative and cytotoxic activity of surface-associated material from periodontopathogenic bacteria. Arch. Oral. Biol. 37:637-644.

9. Meghji, S., B. Henderson, S. Nair, and M. Wilson. 1992. Inhibition of bone DNA and collagen production by surface-associated material from bacteria implicated in the pathology of periodontal disease. J. Periodontol. 63:736-742.

10. Hausmann, E., L. G. Raisz, and W. A. Miller. 1970. Endotoxin: stimulation of bone resorption in tissue culture. Science (Wash. DC). 168:862-864.

11. Meikle, M. C., M. Gowen, and J. J. Reynolds. 1982. Effect of streptococcal cell wall components on bone metabolism in vitro. Calcif. Tissue Int. 34:359364 .

12. Raisz, L. G., C. Alander, G. Eilon, S. P. Whitehead, and K. Nuki. 1982. Effects of two bacterial products, muramyl dipeptide and endotoxin, on bone resorption in organ culture. Calcif. Tissue Res. 34:365-369.

13. Kawata, Y, S. Hanazawa, S. Amano, Y Murakami, T. Matsumoto, $K$. Nishida, and S. Kitano. 1994. Porphyromonas gingivalis fimbriae stimulate bone resorption in vitro. Infect. Immun. 62:3012-3016.

14. Felix, R., H. Fleisch, and P. L. Frandsen. 1992. Effect of Pasteurella multicoda toxin on bone resorption in vitro. Infect. Immun. 60:4984-4988.

15. Lowry, O. H., N. J. Rosebrough, A. L. Farr, and R. J. Randall. 1951 Protein measurement with the Folin phenol reagent. J. Biol. Chem. 193:265-275. 16. Dubois, M., K. A. Gilles, J. K. Hamilton, P. A. Rebers, and F. Smith. 
1956. Colorimetric method for the determination of sugars and related substances. Anal. Chem. 28:350-356

17. Nakashima, K. 1990. Analysis of antigens of Actinobacillus actinomycetemcomitans with monoclonal antibodies. Nippon Shishubyo Gakkai Kaishi 32:71-92.

18. Ey, P. L., S. J. Prowse, and C. R. Jenkin. 1978. Isolation of pure $\mathrm{IgG}_{1}$ $I_{g G}$ and $\operatorname{IgG}_{2 b}$ immunoglobulins from mouse serum using protein A-Sepharose. Biochemistry. 15:429-436.

19. Zanelli, J. M., D. J. Lea, and J. A. Nisbet. 1969. A bioassay method in vitro for parathyroid hormone. J. Endocrinol. 43:33-46.

20. Gitelman, H. J. 1967. An improved automated procedure for the determination of calcium in biological specimens. Anal. Biochem. 18:521-531.

21. Laemmli, U. K. 1970. Cleavage of structural proteins during assembly of the head of bacteriophage $T_{4}$ Nature (Lond.). 227:680-685.

22. O'Farrell, P. H. 1975. High resolution two-dimensional electrophoresis of proteins. J. Biol. Chem. 250:4007-4021.

23. Towbin, H., T. Staehelin, and J. Gordon. 1979. Electrophoretic transfer of gels to nitrocellulose sheets: procedure and some applications. Proc. Natl. Acad. Sci. USA. 76:4350-4354.

24. Koga, T., T. Kusuzaki, H. Asakawa, H. Senpuku, T. Nishihara, and T. Noguchi. 1993. The $64 \mathrm{kDa}$ GroEL-like protein of Actinobacillus actinomycetem comitans. J. Periodontal. Res. 28:475-477.

25. Zahn, R. J. R. Harris, G. Pfeifer, A. Pluckthun, and W. Baumeister. 1993. Two dimensional crystals of the molecular chaperone GroEL reveal structural plasticity. J. Mol. Biol. 229:579-584.

26. Wilson, M., S. Meghji, P. Barber, and B. Henderson. 1993. Biologica activites of surface-associated material from Porphyromonas gingivalis. FEMS Immunol. Med. Microbiol. 6:147-156.
27. Nair, S., Y. Song, S. Meghji, K. Reddi, M. Harris, A. Ross, S. Poole, M Wilson, and B. Henderson. 1995. Surface-associated proteins from Staphylococcus . Bone Miner. Res. $10:$ 734

28. Meghji, S., B. Henderson, and M. Wilson. 1993. High-titer antisera from patients with periodontal disease inhibit bacterial capsule-induced bone breakdown. J. Periodontal. Res. 28:115-121.

29. Dunn, B. E., G. I. Perez-Perez, and M. J. Blaser. 1989. Characterization of Campylobacter pylori proteins by two-dimensional gel electrophoresis and immunoblotting. Infect. Immun. 57:1825-1833.

30. Dunn, B. E., R. Martin Roop, C.-C. Sung, S. A. Sharma, G. I. PerezPerez, and M. J. Blaser. 1992. Identification and purification of a cpn60 heat shock protein homolog from Helicobacter pylori. Infect. Immun. 60:1946-1951.

31. Young, B. D. 1990. Chaperonins and the immune response. Semin. Cell Biol. 1:27-35.

32. Kaufmann, S. H. E., B. Schoel, J. D. A. van Embden, T. Koga, A. WandWurttenberger, M. E. Munk, and U. Steinhoff. 1991. Heat shock protein 60: implications for pathogenesis of and protection against bacterial infections. Immunol. Rev. 121:67-90.

33. Seckinger, P., J. Klein-Nulend, C. Alander, R. C. Thompson, J.-M. Dayer, and L. G. Raisz. 1990. Natural and recombinant human IL-1 receptor antagonists block the effects of IL-1 on bone resorption and prostaglandin production. $J$. Immunol. 145:4181-4184.

34. Nishihara, T., T. Fujiwara, and S. Hamada. 1986. Chemical composition and immunobiological properties of lipopolysaccharide and lipid-associated proteoglycan from Actinobacillus actinomycetemcomitans. J. Periodontal. Res. 21:521-530. 